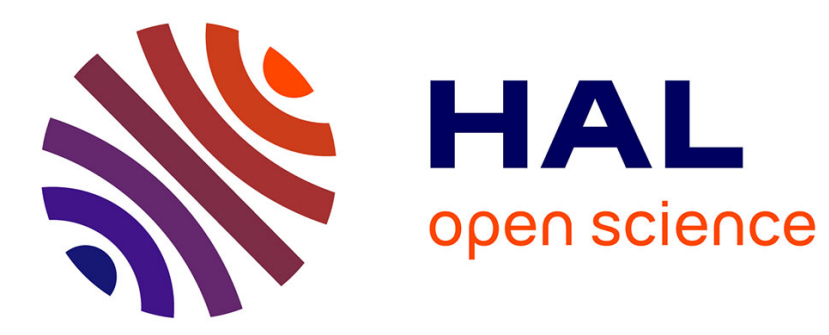

\title{
MIXED VALENT SEMICONDUCTORS : SmB6
}

J. Allen, Roland Martin

\section{- To cite this version:}

J. Allen, Roland Martin. MIXED VALENT SEMICONDUCTORS : SmB6. Journal de Physique Colloques, 1980, 41 (C5), pp.C5-171-C5-176. 10.1051/jphyscol:1980529 . jpa-00219964

\section{HAL Id: jpa-00219964 https://hal.science/jpa-00219964}

Submitted on 1 Jan 1980

HAL is a multi-disciplinary open access archive for the deposit and dissemination of scientific research documents, whether they are published or not. The documents may come from teaching and research institutions in France or abroad, or from public or private research centers.
L'archive ouverte pluridisciplinaire HAL, est destinée au dépôt et à la diffusion de documents scientifiques de niveau recherche, publiés ou non, émanant des établissements d'enseignement et de recherche français ou étrangers, des laboratoires publics ou privés. 
MIXED VALENT SEMICONDUCTORS : $\mathrm{SmB}_{6}$

\author{
J. W. Allen and R. M. Martin \\ Xerox Palo Alto Research Center, Palo Alto, Califomia, USA
}

\begin{abstract}
Résumé.- Nous présentons des arguments expérimentaux et théoriques étayant 1'hypothèse que $\mathrm{SmB}_{6}$ est un semiconduteur de valence uniformément mixte ( $c$ 'est à dire de valence intermédiaire et aux sites équivalents). Il est souligné que les expêriences sur propriétês de transport impliquent que la surface de Fermi est soit extrémement petite, soit non-existante. Des considérations théoriques générale indiquent la possibilité de l'existence d'un gap situé au niveau de Fermi.
\end{abstract}

Abstract.- An overview is given of the experimental and theoretical evidence that SmB is a homogeneously mixed valent semiconductor. It is emphasized that the experimental transport properties imply that the Fermi surface is either extremely small or non-existent, and that rather general theoretical considerations support the possibility of a gap at the Fermi level.

1. Introduction and perspective. - The temperature dependeace of their resistivity divides homogeneously mixed valent rare earth compounds into two groups /1/, those like $\mathrm{CePd}_{3}$ whose low temperature resistivity falls nearly to zero with decreasing temperature $/ 2 /$ and those like $\mathrm{SmB}_{6}$ whose low temperature resistivity becomes very large with decreasing temperature. The members of the former group are correctly regarded as metals. Of the members of the second group, which also includes the gold phase of SmS and the antiferromagnetic phase of TmSe, only $\mathrm{SmB}_{6}$ has ever been modeled as a semiconductor, and the existence of any homogeneously mixed valent insulators has remained a controversial topic in the field of mixed valence. The history of this subject is reviewed briefly in this section.

The temperature dependence of the resistance of $\mathrm{SmB}_{6}$ was reported in 1969 by Menth et al./3/. They found a low temperature resistance rise of a factor of $10^{3}$, exponentially activated with energy $2.5 \mathrm{meV}$ between $4 \mathrm{~K}$ and $15 \mathrm{~K}$, and suggested that $\mathrm{SmB}_{6}$ was a semiconductor in which $\mathrm{Sm}$ was present as $\mathrm{Sm}^{2+}$ and that the activation energy was that required to promote a $4 \mathrm{f}$ electron to the conduction band. This model was disproved in 1970 by Cohen et a1. /4/ who reported that the $\mathrm{Sm}$ isomer shift in the Mössbauer spectrum implied temperature independent valence mixing between $4 \mathrm{~K}$ and $300 \mathrm{~K}$. They suggested that the electron liberated from the $4 f$ shell in valence mixing remained bound to a $\mathrm{Sm}^{3+}$ site at low temperature but did not elaborate on the increased conductivity at higher temperature. In 1971 , Nickerson et a1. /5/ reported the temperature dependence of the resistivity and Hall coefficient in $\mathrm{SmB}_{6}$. They interpreted the data in a three-band model which included a small gap between localized and delocalized states. The localized states had a complex character involving bound $5 \mathrm{~d}$-electrons in order to interpret the Mössbauer and magnetic susceptibility data. They also reported a fairly large linear specific heat which was attributed to carriers in an impurity band. Mott /6/ in 1973 introduced a hybridized $4 \mathrm{f}-5 \mathrm{~d}$ band picture with a gap at the Fermi level. He also attributed the linear specific heat to an impurity band.

Over the same time period, studies of valence mixing due to pressure or alloying in Sm chalcogenides were made. The mixed valence state was obtained from a state of definite semiconductor character, and was generally regarded as metallic, in spite of sma11 low temperature resistance rises in some cases.

With the emergence of mixed valence as a more cohesive field there was a tendency to try to develop a unified model valid for all compounds. Many mixed valent compounds were obviously metallic with large linear specific heats indicative of a large density of states at the Fermi level. Further it became clear that the properties of mixed valent compounds were quite sample-dependent, making it plausible to dismiss the resistivity rise in some of them as an extrinsic effect of some sort. In his 1976 review, Varma /7/ argued against a gap to explain the resistivity rise, citing the non-zero $\gamma$-term in the specific heat, and also the non-zero $\mathrm{T}=0$ magnetic susceptibility. Also in 1976, Kasuya /8/ proposed that the large low temperature resistivity in $\mathrm{SmB}_{6}$ was due to Anderson localization of 
states at the Fermi energy, but that perfect $\mathrm{SmB}_{6}$ would be a metal.

At the 1977 Mixed Valence Conference in Rochester, the metallic model was widely used. Varma /9/ advanced as a general model of the mixed valent ground state the two component Fermi liquid. Kasuya /10/ enlarged on his hopping picture for $\mathrm{SmB}_{6}$ and analyzed the low temperature resistivity with a $\mathrm{T}^{-1 / 4}$ law. Tournier and his co-workers ascribed the low temperature resistance rise in several mixed valent rare earth chalcogenides to Kondo scattering from magnetic impurities $/ 11,12 /$.

The possible existence of homogeneously mixed valent semiconductors was reexamined in a 1978 paper by Allen, Martin, Batlogg and wachter $/ 1 /$, in the context of analyzing the semiconductor-1ike room temperature infrared optical properties of $\mathrm{SmB}_{6}$ and the gold phase of $\mathrm{SmS}$, which had been reported at the Rochester Mixed Valence Conference $/ 13,14 /$. They stated, but without discussion, that the low temperature resistivity in $\mathrm{SmB}_{6}$ was so large it implied a mean free path much less than the lattice constant, and therefore could not be easily dismissed. They also pointed out that specific heat measurements had not then been made on samples of $\mathrm{SmB}_{6}$ with a large low temperature resistivity rise and that measured $\gamma$ 's were smaller in samples with higher low temperature resistivities.

2. Therma1, Electrical and Optical Properties of

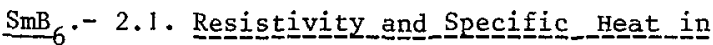
$\mathrm{Sm}_{1-x^{B}-6^{-}}-$Both the resistivity ans specific heat of $\mathrm{SmB}_{6}$ samples have been found to be sample dependent, almost certainly due to variations in stoichiometry, as shown by systematic studies of $\mathrm{Sm}_{1-x^{B}}{ }_{6} / 10 /$. Samples with $x$ closest to zero show the largest low temperature resistivity rise, nearly a factor of $10^{4}$, and the lowest room temperature resistivity. In all samples the resistivity rise saturates below a temperature which is lowest for $\mathrm{x}$ closet to 0 . The first measurements reported on the low temperature specific heat $/ 5 /$ of $\mathrm{SmB}_{6}$ showed it to be not precisely linear with temperature, there being a bump reminiscent of a Schottky anomaly, but $\gamma$ values for two samples could be estimated to be $30 \mathrm{~mJ} / \mathrm{mole}^{2}$, and $80 \mathrm{~mJ} / \mathrm{mole} \mathrm{K}^{2}$. The smaller $\gamma$ was found in the sample with the higher low temperature resistivity. Recently Kasuya and co-workers /15/ have reported a $\gamma$ of $6.8 \mathrm{~mJ} /$ mole $\mathrm{K}^{2}$ for samples with $\mathrm{x}$ nearly zero. In addition, there was around $40 \mathrm{~K}$ a broad peak having the overall features of a Schottky anomaly. The value of $\gamma$ is about twice that of $\mathrm{LaB}_{6}$, but is very much smaller than that which would be expected from a narrow $4 \mathrm{f}$ band at the Fermi leve1 $/ 15 /$. These most recent specific heat results mitigate against a simple heavy Fermi 1iquid picture of the $4 \mathrm{f}$ electrons in $\mathrm{SmB}_{6}$, and suggest the presence of a gap in the electronic states.

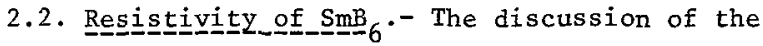
resistivity of nearly stoichiometric $\mathrm{SmB}_{6}$ proceeds most easily with certain principles clearly in mind. For weak scattering in a Fermi liquid, the conductivity $\sigma$ is proportional to the mean free path $L$ of electrons at the Fermi level. In the absence of any disorder, $L$ and the conductivity become infinite as the temperature $\mathrm{T} \rightarrow 0$, the behavior of a perfect Fermi liquid. For a Fermi liquid with static disorder the conductivity is finite as $T \rightarrow 0$, but Mott $/ 16 /$ has argued that there is a lower limit $\sigma_{\min }$ on its value. The existence of this limit is motivated by the expectation that $I$ has a lower limit given by $k_{F} L \sim \pi$, where $k_{F}$ is the Fermi wavevector $/ 17 /$. For a metallic number of carriers, $k_{F}$ is $\sim \pi / a$, so the condition is $L \geq a$, where $a$ is the interatomic spacing. Mott /16/ has identified the regime where disorder would induce $I$ to be much less than the interatomic spacing with disorder-induced Anderson localization $/ 18 /$. If the disorder causing the localization is static, the conductivity at $\mathrm{T}=0$ is expected to be zero, which can be taken to define localization. For $I>0$ the conductivity in states localized by static disorder need not vanish because therma11y activated hopping may occur. At any temperature an experimental conductivity much less than $\sigma_{\min }$ or small enough to imply $\mathrm{L}<$ a for a metallic number of carriers means either that the transport proceeds via localized states or that the number of carriers is much smaller than that of a typical metal.

The value of $\sigma_{\min }$ can be estimated in various ways which give similar order-of-magnitude results. Applying /19/ one of these, known as the unitarity limit $/ 20,21 /$, yields a maximum resistivity $\rho_{u}=$ $544 \mu \Omega \mathrm{cm}$ for $\mathrm{SmB}_{6} / 22 /$, assuming a density of carriers equal to the average number of $\mathrm{Sm}^{3+}$ ions, 
$8.5 \times 10^{21} / \mathrm{cm}^{3}$. It can be shown that $\mathrm{L}$ is approximately the lattice constant for this estimate.

The temperature dependence of the resistivity of a nearly stoichiometric sample of $\mathrm{SmB}_{6} / 22 /$ is shown in Figure 1.

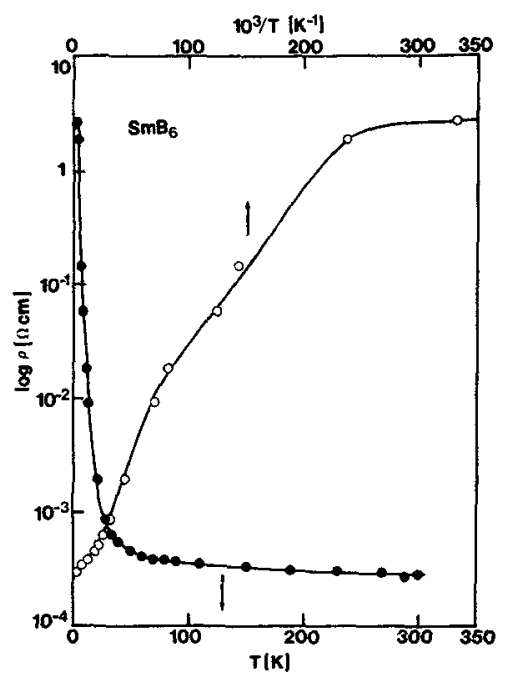

Fig. 1 : Temperature dependence of resistivity of $\mathrm{SmB}_{6}$.

At $300 \mathrm{~K}, \rho$ is $290 \mu \Omega \mathrm{cm}$, not much less than the value of $\rho_{u}$ and implying $L \sim a$. As the temperature decreases the resistivity rises to a value of 2.6 $\Omega \mathrm{cm}$ at $4 \mathrm{~K}$, about 5000 times larger than the value of $\rho_{u}$. For this resistivity, assuming a metallic number of carriers equal to the average density of $\mathrm{Sm}^{3+}$ ions, one deduces /22/ from standard formulas that $\mathrm{L}=2.8 \times 10^{-4} \times \mathrm{a}$, an impossible value. The conclusion is that the resistivity rise must be ascribed to a decrease of carriers relative to that at room temperature or to conduction in states localized by disorder.

A conductivity decrease due to disorder was considered by Kasuya $18,10 /$, who proposed that perfect $\mathrm{SmB}_{6}$ wolld be a metal, but that disorder due to small numbers of Sm vacancies caused the states at the Fermi level to be Anderson localized. Two experimental results disagree with this explanation. One $/ 1 /$ is that deliberate introduction of vacancies increases, rather than decreases, the low temperature conductivity $/ 10 /$. The second, pointed out by Kasuya $/ 15 /$, is that measurements by he and his coworkers of the conductivity down to $15 \mathrm{mK}$ show the conductivity to saturate rather than fall to zero. Assuming this behaviour extends to $\mathrm{T}=0$, it must be concluded that in the samples measured to date hopping transport does not occur at the lowest temperatures and that nonlocalized states exist at the Fermi energy. In the next section the Hall effect is used to estimate carrier densities at high and low temperatures.

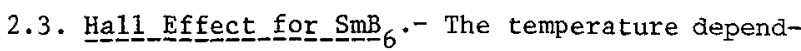
ence of the Hall coefficient $R_{H} / 22 /$ for the sample yielding the data of figure 1 is shown in figure 2.

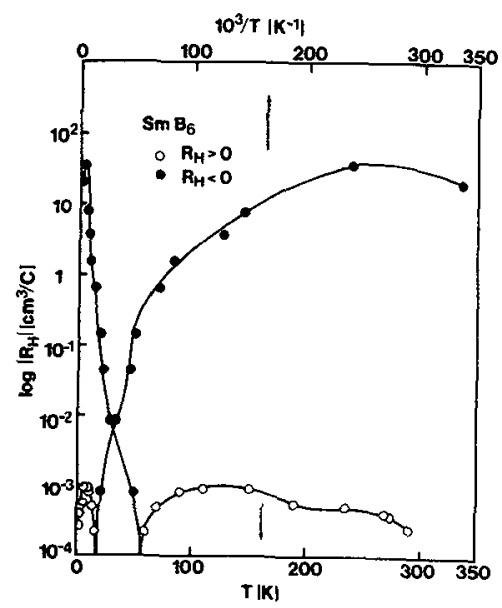

Fig.2 : Temperature dependence of the Hall coefficient of $\mathrm{SmB}_{6}$.

Comparing to data taken on less stoichiometric samples $/ 5 /$, there are significant differences at low temperatures, and the following pattern emerges. As sample stoichiometry improves, the size of the low temperature resistivity rise and the magnitude of the negative $\mathrm{R}_{\mathrm{H}}$ peak increase together, while the temperature at which the resistivity rise saturates and the temperature of the maximum in $\mathrm{R}_{\mathrm{H}}$ decrease together. In contrast to the data of figure 2, for a sample with a resistivity rise of only 1.5 orders of magnitude, the negative peak occurs at $12 \mathrm{~K}$ with a magnitude of only $0.04 \mathrm{~cm}^{3} / \mathrm{C}$ and below $12 \mathrm{~K} \mathrm{R}$ abruptly turns toward zero, becoming positive below $5 \mathrm{~K} / 5 /$. The small positive values of $\mathrm{R}_{\mathrm{H}}$ between $\sim 60 \mathrm{~K}$ and $300 \mathrm{~K}$ show little sample dependence. A sumary of an analysis /22/ of the data of figure 2 is given below.

A detailed analysis of the temperature 
dependence of $R_{H}$ is not possible in the absence of a detailed model of the electronic structure of $\mathrm{SmB}_{6}$. But estimates of carrier concentrations at high and low temperatures can be made unless the Hall and drift mobilities are not comparable, as happens in the anomalous Hall effect or for hopping conductivity. A large anomalous Hall iffect seems unlikely for $\mathrm{SmB}_{6}$ because it does not order magnetically and its magnetic susceptibility is smal1, with a weak temperature dependence compared to that of $\mathrm{R}_{\mathrm{y}}$. The arguments against hopping conductivity have been given above.

Since $R_{H}$ changes sign with temperature variation, the simple two parabolic-bands model has been used to estimate carrier densities. It can be shown $/ 22 /$ that in this model upper bounds on the densities of holes and electrons follow simply from placiag a lower limit on the mobility of the carrier which does not determine the sign of $\mathrm{R}_{\mathrm{H}}$. This limit was chosen as that corresponding to the resistivity $\rho_{u}$, and is equal to $1.35 \mathrm{~cm}^{2} / \mathrm{v} \mathrm{sec}$. For room temperature the analysis gives carrier densities $\sim 9 \times 10^{21} / \mathrm{cm}^{3}$, and mobilities $\sim 1.8 \mathrm{~cm}^{2} / \mathrm{V} \mathrm{sec}$; at $4 \mathrm{~K}$ the carrier densities are less than $25 \times 10^{17}$, $\mathrm{cm}^{3}$ and the mobilities are in the range $2-40 \mathrm{~cm}^{2}$ / $\mathrm{V}$ sec.

The room temperature carrier density is very close to the average number of $\mathrm{sm}^{3+}$ sites, and the mobility and resistivity are close to the unitarity limit values, leading to a picture of $\mathrm{SmB}_{6}$ at room temperature as a poor metak. As the tempexature decreases the number of carriers decreases by a factor of $\sim 10^{4}$, causing the conductivity to decrease by a factor of $\sim 10^{4}$. Such behavior is compatible with a small gap semiconductor model for possibly a serimetal model with a very narrow density-of-states minimum), and activation energies of about $1.8 \mathrm{meV}$ and $2.7 \mathrm{meV}$ can be deduced from, respectively, plots of $\log \left|R_{H}\right|$ and $\log \rho$ vs. $1 / T$, in the temperature range $4 \mathrm{~K}-13 \mathrm{~K}$. A gap the order of magnitude of these activation energies is consistent with the Schottky type specific heat anomaly, and with the bad metal behavior at room temperature, where $\mathrm{kT}$ is about 10 times larger than the activation energy $/ 6 /$.

2.4. The Residiual_Conductivivity. - As emphasized by Kasuya /15/ it is important to assess whether the residual conductivity is consistent with estimates of minimum metallic conductivity. Using /22/ the low temperture carrier density deduced from the Hall effect to estimate the characteristic length in an expression given by Mott /23/ for $\sigma_{\min }$ yields $\sigma_{\min }$ $=5.7\left(\Omega \mathrm{cm}^{-1}\right.$, about 15 times the observed conductivity of $0.38(\Omega \mathrm{cm})^{-1}$. Given the general difficulty of assessing $\sigma_{\min } / 23 /$ and the lack of detailed knowledge of the states at the Fermi energy, it is by no means clear that a factor of 10 is cause for alarm.

Kasuya /15/ has compared the observed conductivity with a much larger estimate of $\sigma_{\text {min }}$, appropriate for a metallic number of carriers, and argued that a novel model of the ground state is required to evade the conflict. To this end, he has proposed that a gap exists at the Fermi energy due to the formation of a Wigner lattice. In the absence of disorder the conductivity would be infinite at $T=0$ due to phase slippage of the lattice, Disorder pins the lattice and reduces the conductivity to the observed value. Again it is appropriate to point out that the experimental fact that the conductivity increases with degraded stoichiometry mitigates generally against any models in which defects reduce otherwise infinite conductivity. Also Kasuya's estimate of $\sigma_{\min }$ is unnecessarily large, as shown above.

Nonetheless, the issue of the residual conductivity and especially its relation to the residual linear specific heat requires further experimental and theoretical consideration.

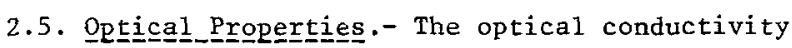
$/ 1 /$ of $\mathrm{SmB}_{6}$ for temperatures between $300 \mathrm{~K}$ and $4 \mathrm{~K}$ is that of a metal at photon energies greater than $\sim 0.5 \mathrm{eV}$, and there is a temperature independent $/ 13 /$ plasma edge in the optical reflectivity at $\sim 1.5 \mathrm{eV}$. It is to be emphasized that a sma1l gap at the Fermi level will not affect the high energy dielectric response, and that the energy of the reflectivity edge has no automatic relation to the carrier density involved in d.c. transport. Assertions to the contrary $/ 24 /$ are incorrect. At room temperature the optical properties of $\mathrm{SmB}_{6}$ between 0.02 and $0.5 \mathrm{eV}$ are insulator-like in that the real part of the dielectric constant becomes large and positive at low energy, but this behavior can be accounted for in the bad-metal model valid at high temperatures $/ 1 /$. Low temperature, low 
frequency spectroscopy in the meV range, which could reveal details of the energy levels near the Fermi energy, remains to be done.

3. Theory of the Hybridization Gap.- Mott's proposal $/ 6 /$ of a $4 f-5 d$ band model with a hybridization gap at the Fermi level left open two questions, whether simple well-defined bands are appropriate to describe the interacting $4 \mathrm{f}$ electrons, and if so, whether a gap is likely to occur for a realistic band structure. Both of these questions have been addressed in a recent theoretical study $125 /$. There is space here to summarize only the most salient points of the theory, as follows.

(a) $\mathrm{SmB}_{6}$ is cubic with $\mathrm{Sm}$ ions at sites with full $o_{h}$ symmetry. Symmetry arguments show that there is an isolated, lowest-energy, fermion hole excitation in which an electron is removed from a $\mathrm{Sm}^{2+}$ ion in its ground $\mathrm{J}=0$ state to create a $\mathrm{Sm}^{3+}$ ion in the lowest energy state split off from the ground ${ }^{6} \mathrm{H}_{5 / 2}$ configuration by the cubic crystal field. This excitation is a doublet, transforming as $\Gamma_{7}-$ in $O_{h}$, and a single such excitation results in a welldefined band.

(b) Band calculations for $\mathrm{LaB}_{6} / 26 /$ show that in $\mathrm{SmB}_{6}$ the Fermi leve1 will lie in an isolated band, which is orbitally nondegenerate except at $\Gamma$ and which has its minimum at the $\mathrm{X}$-point. For homogeneous mixed valence, the Fermi level must also intersect the $4 f$ excitation described in (a).

(c) It happens that this band and the $4 f$ excitation have the same symmetry throughout the Brillouin zone, except at $\Gamma$. Were there not strong Coulomb interactions of the dense number of $f$ holes with each other or with the band electrons, then there would be a gap if the $4 \mathrm{f}$ band had zero or positive dispersion from $\Gamma$ to $X$, and counting electrons shows that the Fermi level would lie in the gap. This situation is shown in Figure $3(a)$.

(d) Including the Coulomb interactions the problem for the states closest to the Fermi level is equivalent to a two-band Hubbard model in which the site occupations for the narrow $f$-band are either one or two. Solving for the one-particle Green's function using the decoupling approximations of Hubbard III $/ 27,28 /$ shows that, if the narrow band occupation $n_{f}$ is not too close to one, the gap in the density of states remains, even though two major changes in the simple band picture occur. These changes, shown in figure $3(\mathrm{~b})$, are that a portion of the occupied density of states is split off to lower energies by $U$, the $4 \mathrm{f}$ hole-hole Coulomb interaction, and that the f-states suffer strong Coulomb scattering which decreases the gap and induces uncertainty in $k$. Very near the band gap this uncertainty is an artifact of the calculation, but away from the gap it is real. The details of the conditions for gap existence and other features of the solution are described elsewhere/25/.

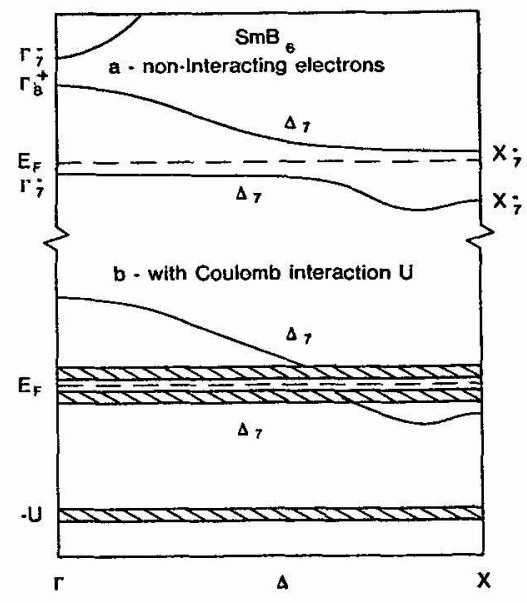

Fig. 3 : Schematic band diagram for $\mathrm{SmB}_{6}$. (a) Simple bands for lowest energy Sm $f^{6} \leftrightarrow f^{5}$ transition and conduction band as determined in Ref. /26/. (b) Including large $\mathrm{f}-\mathrm{f}$ Coulomb interaction, as discussed in text.

(e) If there is an even integral number of electrons per atom, then the Fermi level remains in the gap for all cases in which the gap exists, in spite of the altered density of states. That this is so can be regarded as a consequence of the Luttinger theorem /29/ which states that turning on Coulomb interactions does not alter the volume enclosed by the Fermi surface provided that there is no change of symmetry and that no states cross the Fermi energy. The gap itself is most easily understood by considering the mixed valent states as an analytic continuation from the integral valent state in which the Fermi level is in a gap between the filled $4 \mathrm{f}^{6}$ states and the unoccupied broad $5 \mathrm{~d}-$ band. That the integral valent state for $\mathrm{Sm}$ is the nondegenerate $4 f^{6} \mathrm{~J}=0$ configuration is of obvious central importance in the application of these two general ideas.

4. Otwer Compounds.- Other compounds of interest 
are $\mathrm{SmS}$, whose mixed valent phase shows a modest low temperature resistivity increase /30/, and Tmse, whose resistivity is high in its antiferromagnetic phase, but low in its ferromagnetic and paramagnetic phases $/ 31,32 /$. Band calculations for insulating SmS show that electrons liberated from the $4 \mathrm{f}$ shell wi11 occupy a singly degenerate band with a minimum at the $x$-point. This band has the same symmetry as the $f$-band except at the $\Gamma$ and $X$ points, implying the possibility of a very small Fermi surface. TmSe presents additional complications because the mixed valence state involves $\mathrm{Tm}^{2+}$ $\left(4 \mathrm{f}^{13}\right)$ with ${ }^{2} \mathrm{~F}_{7 / 12}$ ground state and $\mathrm{Tm}^{3+}\left(4 \mathrm{f}^{12}\right)$ with ${ }^{3} \mathrm{H}_{6}$ ground state, each of which is degenerate. The results of Ref./25/ suggest that a gap is unlikely in a ferromagnetic mixed valence state, whereas a gap is not obviously precluded in the antiferromagnetic phase.

5. Summary.- In summary, the folowing points should he emphasized for $\mathrm{SmB}_{6}$.

(a) The experimental properties imply a Fermi surface that is either extremely small or nonexistent, and are completely inconsistent with a model of the $4 f$ electrons as a heavy Fermi liquid of metallic density.

(b) Rather general theoretical considerations show that homogeneously mixed valent $\mathrm{SmB}_{6}$ can have a gap at the Fermi level.

Acknowledgments.- We gratefully acknowledge P. W. Anderson, B. Batlogg, B. R. Coles, S. Doniach, R. J. Elliott, C. Herring and P. Wachter for very helpful conversations.

\section{References}

/1/ Alien, J.W., Martin, R.M., Batlogg, B., and Wachter, P., App1. Phys. 49 (1978) 2078.

12) Scoboria, P., Crow, J.E., and Mihalisin, T., J. App 1. Phys. 50 (1979) 1895.

/3/ Menth, A., Buehler, E., and Geballe, T.H., Phys. Rev. Lett. 22 (1969) 295.

14/ Cohen, R.L., Eibschutz, M., and West, K., Phys. Rev. Lett. 24 (1970) 383 .

/5/ Nickerson, J.C., White, R.M., Lee, K.N., Bachman, R., Geballe, T.H., and Hull, G.W.,Jr., Phys. Rev. B33 (1971) 2030.

16/ Mott, N.F., Philos. Mag. 30 (1973) 403.

17/ Varma, C.M., Rev. Mod. Phys. 48 (1976) 219.
18/ Kasuya, T., J. Phys. 37 Colloq. (1976) p.C4261 .

19/ See, e.g, Varma, C.M., in Valence Instabilities and Related Narrow Band Phenomena, edited by Parks, R.D., (Plenum, New York, 1977) p.201.

/10/ Kasuya, T., Kojima, K., and Kasaya, M., ibid, P. 137 .

/11/ Berger, A., Bucher, E., Haen, P., Holtzberg, F., LaPierre, F., Penny, T., and Tournier, R. ibid, p. 491 .

/12/ Chenevas-Paule, A., Haen, P., LaPierre, F., Tournier, R., and Vieux-Rochas, L., ibid, p. 513 .

/13/ Allen, J.W., ibid, p. 533.

/14/ Batlogg, B., Wachter, P., ibid, p.537

/15/ Kasuya, T., Takegahara, K., Fujita, T., Tanaka, T., and Bannai, E., J. Phys. Colloq. 40 C5 (1979) p. C5-308.

/16/ Mott, N.F., Metal-Insulator Transitions (Taylor and Francis, LTD, Lendon,) 1974 p.27.

/17/ Ioffe, A.F., and Rege1, A.R., Prog. Semicond. 4 (1960) 283 .

/18/ Anderson, P.W., Phys. Rev. 109 (1959) 1492.

119/ Andres, K., Graebner, J.E., and Ott, H.R., Phys. Rev. Lett. 35 (1975) 1779.

120/ Friede1, J., Nuovo Cimento 7 Supp1. (1958) 287.

121/ Schrieffer, J.R., J. Appl. Phys. $\underline{38}$ (1967) 1143.

$122 /$ Allen, J.W., Batlogg, B., and Wachter, P., Phys. Rev. (1980), to be published.

/23/ Mott, N.F., Metal-Insulator Transitions (Taylor and Francis, LTD, London) 1974 p. 33

/24/ Keller, R., Guntherodt, G., Holzpfel, W.B., Dietrich, M., and Holzberg, F., Solid State Commun. 29 (1979) 753.

/25/ Martin, R.M., and Allen, J.W., J, Appl. Phys. 50 (1979) 7561 .

126/ Hasegawa, A., and Yanase, A., J. Phys. F7 (1977) 1245 .

127/ Hubbard, J., Proc. R. Soc. A281 (1964) 401.

128 / The method is very similar to recent work by Sakai, Seki and Tachiki (J. Phys. Soc. Japan 45 (1978) 1465), although the underlying physical model is quite different.

129/ Luttinger, J.M., Phys. Rev. 119 (1960) 1153.

/30/ Bader, S.D., Phillips, N.E., and McWhan, D.B., Phys. Rev. B7 (1973) 4686.

131/ Holtzberg, F., Penney, T., and Tournier, R., J. Phys. Colloq. C5 (1979) p. C5-314.

132/ Batlogg, B., ott, H.R., Kaldis, E., Thoni, W., and Wachter, P., Phys. Rev. B19, 247 (1979). 